\title{
In Differential Diagnosis of Dementia Application of Easy Z-score Imaging System in Single Photon Emission Computed Tomography Brain Perfusion Scan
}

\author{
Nasreen Sultana ${ }^{1, ~ *, ~ H i r o s h i ~ M a t s u d a ~}{ }^{2}$, Azmal Kabir Sarker ${ }^{3}$, Jasmine Ara Haque ${ }^{1}$, Rafiqul Islam ${ }^{4}$ \\ ${ }^{1}$ National Institute of Nuclear Medicine and Allied Sciences (NINMAS), BSMMU Campus Dhaka, Bangladesh \\ ${ }^{2}$ Integrative Brain Imaging Center, National Center of Neurology and Psychiatry, Tokyo, Japan \\ ${ }^{3}$ Institute of Nuclear Medicine and Allied Sciences, Mitford, SSMC Campus, Dhaka, Bangladesh \\ ${ }^{4}$ Neurology, BSM Medical University, Dhaka, Bangladesh
}

Email address:

nasreeninm@yahoo.com (N. Sultana),ninmas05@gmail.com (N. Sultana)

${ }^{*}$ Corresponding author

\section{To cite this article:}

Nasreen Sultana, Hiroshi Matsuda, Azmal KabirSarker, Jasmine Ara Haque, Rafiqul Islam. In Differential Diagnosis of Dementia Application of Easy Z-score Imaging Systemin Single Photon Emission Computed Tomography Brain Perfusion Scan. American Journal of Psychiatry and Neuroscience. Vol. 7, No. 4, 2019, pp. 100-107. doi: 10.11648/j.ajpn.20190704.14

Received: October 20, 2019; Accepted: November 6, 2019; Published: November 12, 2019

\begin{abstract}
Application of easy Z score imaging system (eZIS) on brain perfusion SPECT images for quantitative evaluation of $\mathrm{rCBF}$ has attempted to explore the differential diagnosis of dementia with various degenerative diseases and to evaluate discrimination of early Alzheimer's disease (AD) from other types of dementia. This retrospective study was done with various degenerative diseases that underwent brain perfusion SPECT using $99 \mathrm{mTc}$ ethyl cysteinate dimer were included. Brain perfusion SPECT was performed in 58 patients $(\mathrm{M} / \mathrm{F}=48 / 10)$ with mean age of $61.2 \pm 10.4$ years (41-90). Decreased $\mathrm{rCBF}$ was observed in the area suspected early $\mathrm{AD}$ in 20 cases (34\%) and in the frontal gyrus and insula in 10 cases $(17 \%)$. And decreased $\mathrm{rCBF}$ was observed in occipital lobe and, precuneus, gyrus and posterior cingulate cortex in 8 cases (14\%). Discrete areas of decreased $\mathrm{rCBF}$ were seen in 14 cases $(24 \%)$ and the rest of 6 cases $(11 \%)$ showed normal rCBF. Clinically suspected $\mathrm{AD}$ patients were divided into two groups on basis of MMSE score (group A, mild stage of AD, MMSE $=20-26$ and group $\mathrm{B}$, moderate to severe stage of $\mathrm{AD}, \mathrm{MMSE}=10-19)$. Mean MMSE and mean $\mathrm{Z}$ scores were $22.9 \pm 1.11$ and $1.01 \pm 0.35$ in group $\mathrm{A}(\mathrm{n}$ $=12$ ) while $17.56 \pm 0.96$ and $2.62 \pm 0.59$ in group $B(n=8)$. There was negative correlation between MMSE and eZIS score; $r=-$ 0.69 in group $A(p=0.002) ; r=-0.58$ in group $B(p=0.019)$ and $r=-0.93$ overall $(p=0.000)$. Quantification of $r C B F$ with eZIS score was well associated with clinical MMSE scoring in this study group with clinical likelihood of having AD. In rest of the cases, SPECT findings and surface view analysis by eZIS, the affected area of decreased rCBF were consistent with clinically suspected different types of degenerative disease manifesting dementia.
\end{abstract}

Keywords: eZIS, Alzheimer's Disease, Brain Perfusion, SPECT

\section{Introduction}

Brain single photon emission computed tomography (SPECT) is a well-established and reliable method to assess brain function through measurement of regional cerebral blood flow (rCBF). It can be used to define a patient's pathophysiological status when neurological symptoms cannot be explained by anatomical neuroimaging findings. SPECT is a technique that produces tomographic images ofthe three-dimensional distribution of a radiopharmaceutical. Applied to the brain; this technique can be used to measure regional cerebral perfusion. As such the true role of imaging is often imaging of the brain in patients with suspected neurodegenerative conditions is common and challenging, as in patients with indirect and confusing signs and symptoms, the imaging findings are also subtle and equivocal. Significant progress in diagnostic accuracy has beenmade using positron emission tomography (PET) andsingle-photon emission computed tomography (SPECT) in the diagnosis of 
Alzheimer's disease (AD). Computer assisted analysis using voxel-based morphometry (VBM) [1] from statistical parametric mapping (SPM) [2] for magnetic resonance imaging (MRI) and three-dimensionalstereotactic surface projection (3D-SSP) [3-5] or the easy Z-score imaging system (eZIS) [6-10] for PET/SPECT has been developed. Therefore, increasingly unbiasedand dependable information on abnormalities in regional cerebral blood flow (rCBF) can be achieved. Individual neurodegenerative diseases have relatively specific characteristic clinical features [2]. The most common neurodegenerative disorders that present with dementia are Alzheimer's disease (AD), dementia with Lewy bodies (DLB) and frontotemporal lobar degeneration (FTLD) eZIS allows computer assisted statistical analysis of brain perfusion SPECT images. Voxel based analysis performed by using a Z-score map calculated from comparison of patient's data with the control data base in same manner as in a 3DSSP method. It assesses severity of regional cerebral blood flow ( $\mathrm{rCBF}$ ) decreased in a specific region of $\mathrm{AD}$, extent of area of decreased $\mathrm{rCBF}$ and ratio of area of decreased $\mathrm{rCBF}$ to that of whole brain. In our study we consider the $\mathrm{Z}$ score severity for differential diagnosis as well as surface view analysis for affected brain area.

In Bangladesh, ${ }^{18} \mathrm{~F}$-fluorodeoxyglucose positron emission tomography $\left({ }^{18} \mathrm{~F}\right.$-FDG PET) for the diagnosis of dementia is not routinely practiced as it is expensive and not reimbursed yet by the our existing health insurance system, and thus the more widely available MRI is primarily used for imaging diagnosis of neurodegenerative disease. PET is more sensitive than SPECT in diagnosing early AD and other type of dementia, but SPECT offers the advantages of lower cost than PET and ease of entrance. A specific decreased in $\mathrm{rCBF}$ in the posterior cingulate gyrus, precuneus and parietal cortices in the very earlystage of $\mathrm{AD}$ has been detected by SPECT [6-9]. eZIS is a method of statistical analysis for the automated diagnosisof brain perfusion SPECT images which can be used to investigate the rCBF objectively and easily [610]. SPECT combined with eZIS has been applied in clinical practiceat NINMASas a valuable early diagnostic method in patients with clinically suspected $\mathrm{AD}$ and to findout the differential diagnosis of dementia since 2017. Waragai et al. revealed that eZIS analysis of SPECT images could be convenient for the early and differential diagnosis of patients with neurodegenerative disease, including dementing diseases, $\mathrm{AD}$, frontotemporal dementia (FTD), dementia with Lewy bodies (DLB) [10]. In patients with very early AD, rCBF decreased significantly in the posterior cingulate cortex, precuneus and parietal cortex whereas eZIS analysis in patients with DLB showed decreases in $\mathrm{rCBF}$ extending from the precuneus gyrus to the occipital cortex. These findings suggest neurodegenerative changes in DLB. FTD is also common neurodegenerative dementia. We demonstrated decreased $\mathrm{rCBF}$ in the frontal cortex, insula, temporal cortex andorbitofrontal cortex, regions which are related to disruptivebehavioral symptoms with relative sparing of theposterior cortex in patients with FTD [10]. Matsuda et al. have developed an automatedsystem of analysis using eZIS with incorporation of avolume of interest (VOI) related to early $\mathrm{AD}$, and show edits high performance in selective patients with veryearly $\mathrm{AD}$ from age-matched healthy volunteers [8]. The purpose of the current study was to evaluate thissystem as an adjunct for the early diagnosis of $\mathrm{AD}$ and other types of dementia.

\section{Aims \& Objective}

Application of eZIS on brain perfusion SPECT images for quantitative evaluation of $\mathrm{rCBF}$ has endeavored to explore association of MMSE scoring with clinically suspected AD and to evaluate computer-assisted automated discrimination of early Alzheimer'sdisease (AD) from other types of dementia. This study has also attempted to explore the association of MMSE scoringand $\mathrm{Z}$ score in patients with clinical likelihood of having Alzheimer's disease (AD).

\section{Methods}

This retrospective study was done at NINMAS, BSM Medical University, Dhaka. Patients with various neurodegenerative diseases and on basis of clinical information, anatomical imaging and MMSE scorewho underwent brain perfusion SPECT using Tc-99m ethyl cysteinate dimer (ECD) from February 2017 to June 2019 were included in this study. Informed written consent was taken from each patient prior brain SPECT study. eZIS version 3 (Mastuda et al.) was applied to brain perfusion SPECT DICOM data.

\subsection{Patient Preparation}

In first appointment all information regarding the brain SPECT procedure, pre - preparation were discussed with patient and inform content was taken. Before arrival, patients were instructed to avoid, if possible, caffeine, alcohol, or other drugs known to affect cerebral blood flow (CBF). Before the SPECT scanning was performed, all subjects had an intravenous line established.

\subsection{Study Population \& Technique}

\subsubsection{Subjects}

Patients with clinical diagnoses of dementia were included in this study, which was conducted with the approval of the MREC (MedicalResearch Ethical Committee) of NINMAS. Disease onset was consideredto be the time when symptoms first appeared. Brain perfusion SPECT was performed in 58 patients $(\mathrm{M} / \mathrm{F}=48 / 10)$ with clinically diagnosed various neurodegenerative disease with mean age of $61.2 \pm 10.4$ years (41-90) and mean duration since clinical onset of 10.3 \pm 4.7 months (6-24). The patients' demographic characteristics are listed in Table 1.

The clinical diagnosis was established based ona detailed medical history, physiological and neurological examinations, the results of routine laboratory investigations, and MRI of the brain. 


\subsubsection{Brain SPECT Procedure}

An intravenous line was established in all subjects beforeSPECT imaging was performed. While lying supine with eyes closed in a dimly lit quiet room, each subject received an intravenous.

Injection of $600 \mathrm{MBq}$ of ${ }^{99 \mathrm{~m}} \mathrm{Tc}-\mathrm{ECD}$. Ten minutes after thisinjection, brain SPECT was performed with a doubleheadedgamma camera (Duel Head Gamma camera, Model: Symbia Evo Excel (Siemens, USA) equipped with lowenergy and high-resolution parallel-holecollimators. For each camera, projection data were obtained in a $128 \times 128$ matrix through $360^{\circ}$ rotation at steps of $2.8^{\circ}$ for 20 sper view. Filtered back-projection using a Butterworth and Rampfilter was used for SPECT image reconstruction. Reconstruction \& display general tomographic data via OSEM iterative reconstruction method. Attenuation correction by Chang's method. Table 2 depicted the Brain perfusion SPECT findings among the study people.

\subsection{SPECT Image Analysis Using eZIS}

SPECT images of all patients and all diseases were standardized anatomically with an original 99m Tc-ECD template using eZIS. An averaged SPECT image for each disease was then createdfrom these anatomically standardized images. A Z-scoremap for the averaged SPECT image for each disease was obtainedby comparing SPECT images of age-matched healthy volunteerswith the mean and standard deviation for each voxel obtainedafter anatomical standardization and voxel normalization toglobal mean values using the following equation: $Z$-score $=($ [controlmean $]$ - [individual value])/(control SD). The Z-score mapswere displayed by overlay onto topographic sections and projection, with an averaged Z-score obtained from a depth of 14 mmto the surface through rendering of an anatomically standardized MRI template. This voxel based analysis is performed by using a Z-score map calculated from comparison of patient's data with the control data base in same manner as in a 3D-ssp method. SPECT using e ZIS was performed among all patients SPECT DICOM data developed by Matsuda et al. [8]. We assessed decreased $r$ $\mathrm{CBF}, \mathrm{Z}$ score in clinically suspected AD patients and surface view mode in various section of brain image. Three indicators were used for characterizing the rCBF. Decreasesusing a Z-score in the VOI, which was defined as the regionrelated to $\mathrm{AD}$ in early $\mathrm{AD}$, were automatically determined asfollows. First, the severity of the decrease in $\mathrm{rCBF}$ in the specific region showing a decrease in $\mathrm{rCBF}$ in early $\mathrm{AD}$ was obtained fromthe averaged positive $\mathrm{Z}$-score in the VOI. Second, the extent of theregion showing a significant decrease in $\mathrm{rCBF}$ in the VOI was obtained; that is, the percentage of coordinates with a Z-score exceedingthe threshold value of 2.0 was determined. Third, theratio for each region showing a significant decrease in $\mathrm{rCBF}$ in theVOI relative to the region showing a significant decrease in rCBFin the whole brain was obtained; this ratio ( $\mathrm{rCBF}$ ratio) indicates the specificity of the decrease in $\mathrm{rCBF}$ in the VOI compared withthe whole brain. According to Matsuda et al. The accuracies of severity, extent and the $\mathrm{rCBF}$ ratio in discriminating betweenhealthy controls and patients with early $\mathrm{AD}$ are 85,86 , and $80 \%$, respectively, based on cutoff values of $1.19,14.2 \%$, and 2.22 , respectively [8].

Table 2 shown the brain SPECT perfusion findings with decreased $\mathrm{rCBF}$ among the study population.

\section{Results}

Brain perfusion SPECT was performed in 58 patients $(\mathrm{M} / \mathrm{F}$ $=48 / 10$ ) with clinically diagnosed various neurodegenerative diseases with mean age of $61.2 \pm 10.4$ years $(41-90)$ and mean disease duration since clinical onset of $10.3 \pm 4.7$ months $(6$ 24). Decreased rCBF was observed in the tempero-parietal region, posterior cingulate gyri and precuneus in suspected early $\mathrm{AD}$ in 20 cases $(34 \%)$ and in 10 cases $(17 \%)$ in the frontal gyrus and insula. And decreased rCBF was observed in 8 cases $(14 \%)$ in occipital lobe, precuneus and parietal cortex. Discrete areas of decreased rCBF were seen 14 cases $(24 \%)$ and the rest of 6 cases $(11 \%)$ showednormal rCBF. Figures 1, 2, 3, 4, $5 \& 6$ shown the surface view of decreased rCBF, severity, extent and ratio after application eZIS in patients DICOM data. Clinically suspected AD patients were divided into two groups on basis of MMSE score (group A, mild stage of $\mathrm{AD}, \mathrm{MMSE}=20-26$ and group $\mathrm{B}$, moderate stage of $A D, M M S E=10-19)$. Mean MMSE and mean $Z$ scores were $22.9 \pm 1.11$ and $1.01 \pm 0.35$ in group $A(n=12)$ while $17.56 \pm 0.96$ and $2.62 \pm 0.53$ in group $B(n=8)$. There was negative correlation between MMSE and eZIS Z-score; $r$ $=-0.69$ in group $\mathrm{A}(\mathrm{p}=0.002) ; \mathrm{r}=-0.58$ in group $\mathrm{B}(\mathrm{p}=$ $0.019)$ and $r=-0.93$ overall $(p=0.000)$. Figure 7 depicted the scatter plot of negative correlation between MMSE and Z-score severity from eZIS, in patients with clinical likelihood of having $\mathrm{AD}$. The severity, extent and ratio of the decreased $\mathrm{rCBF}$ in suspected $\mathrm{AD}$ patients were significantly greater than in other types of neurodegenerative disease manifesting dementia.

Table 1. Demographic and clinical data of subjects.

\begin{tabular}{ll}
\hline Parameters & Number \\
\hline No of patients & 58 \\
Age range & $(41-90)$ years \\
Mean Age & $61.2 \pm 10.4$ years \\
M/F & $48 / 10$ \\
Main disease duration (m) & $10.3 \pm 4.7$ months $(6-24)$ \\
Mean MMSE for suspected AD patients & $22.9 \pm 1.11$ group A $(\mathrm{n}=12)$ \\
\end{tabular}


Table 2. Brain SPECT findings.

\begin{tabular}{lll}
\hline Cerebral Area & Findings & No of patients \\
\hline Frontal region & Decreased radiotracer uptake & $10(17 \%)$ \\
Temporo-parietal region & & $20(34 \%)$ \\
Posterior cingulate gyri & Decreased radiotracer uptake & \\
Precuneus & & $08(14 \%)$ \\
Occipital lobe, precuneus, parietal cortex & Decreased radiotracer uptake & $14(24 \%)$ \\
Discrete areas & Decreased radiotracer uptake & $06(11 \%)$ \\
\hline
\end{tabular}

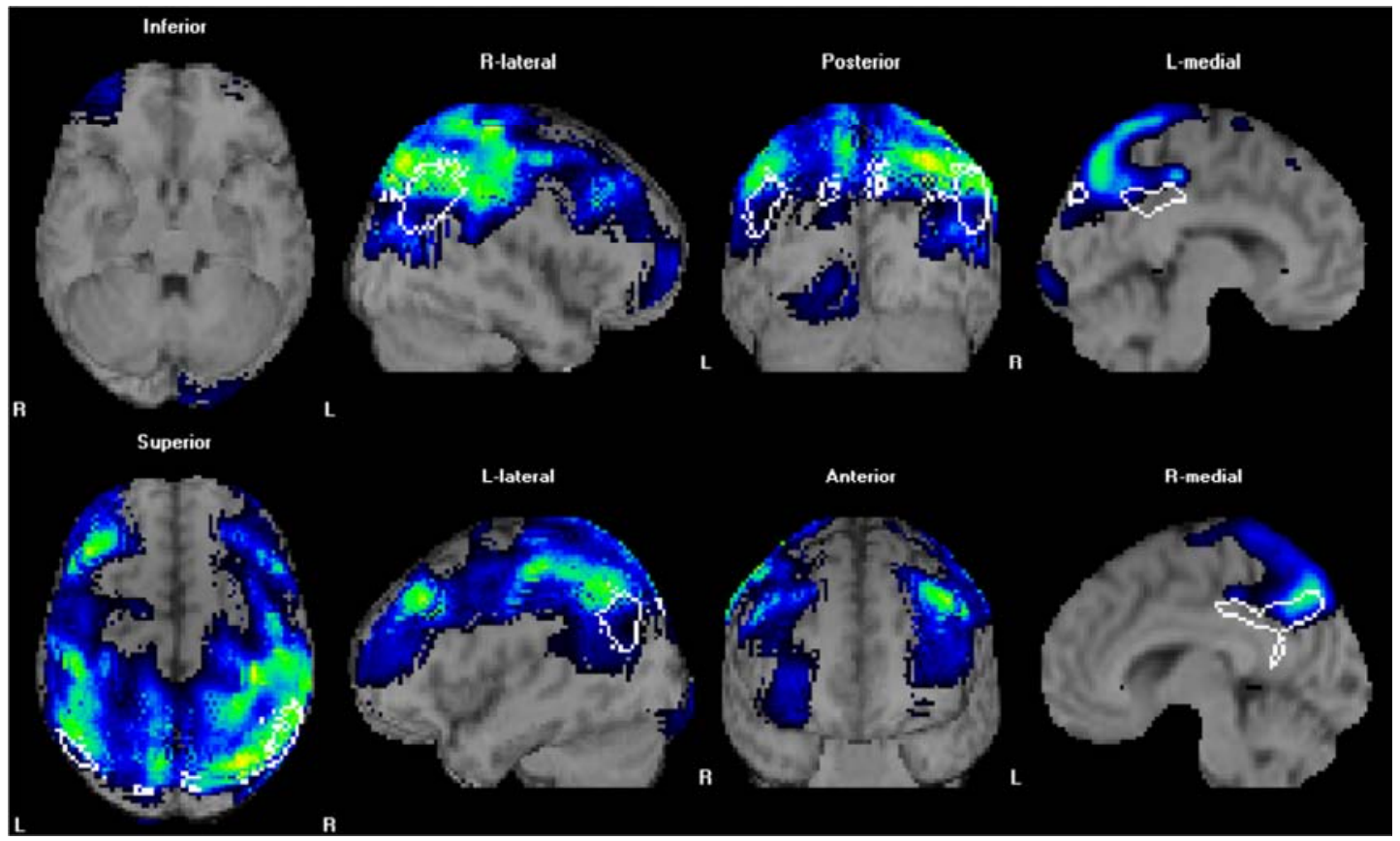

Figure 1. A 77-years-old male, MMSE 24, Clinically suspected AD with a 14 month disease duration had decreased $r C B F$ in the parietal cortex and the posterior cingulate cortex. The severity index, extent and $r C B F$ ratio were severity: 2.42, Extent: $56.60 \%$, Ratio: 3.6 respectively.

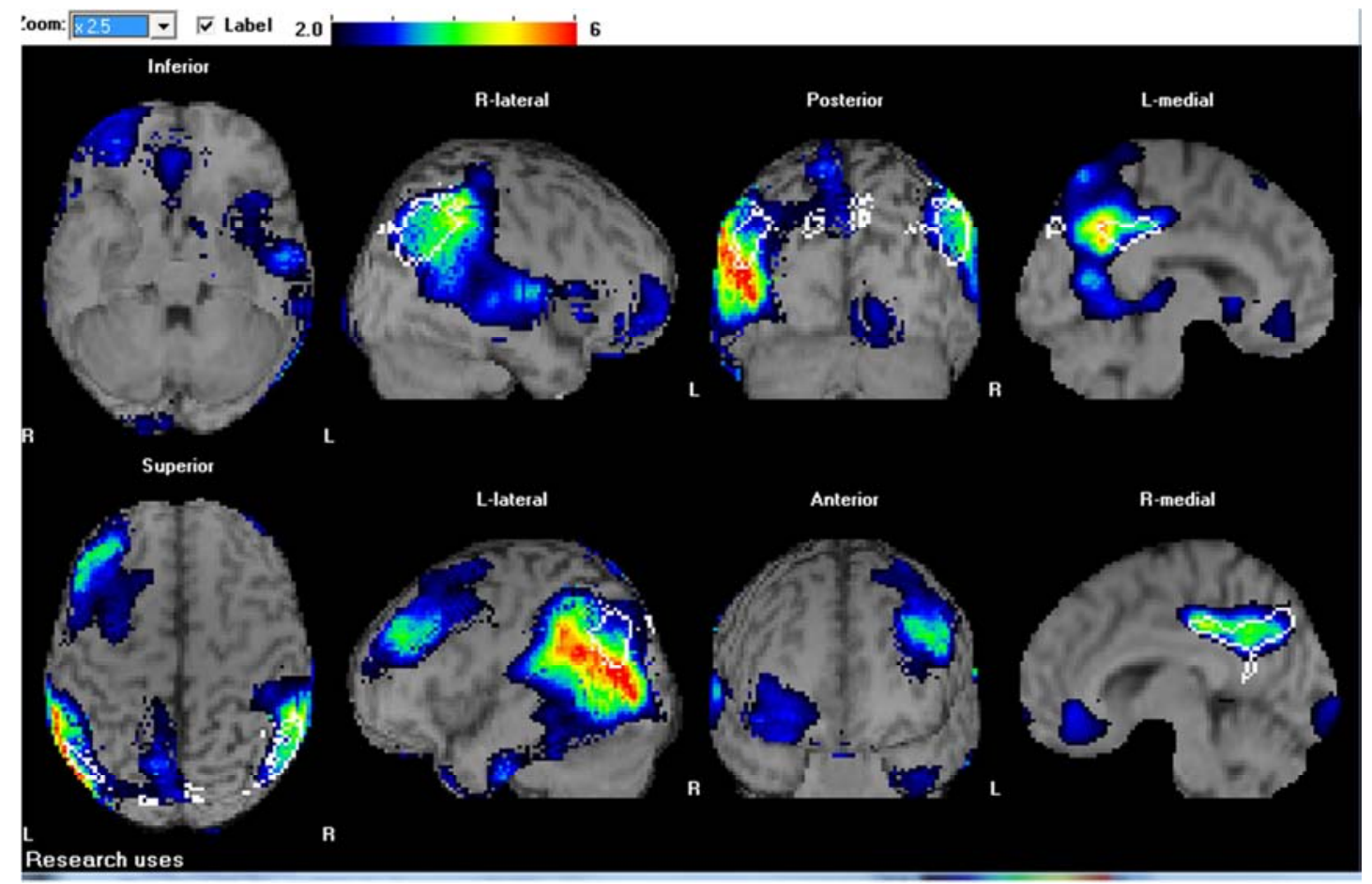

Figure 2. A 67-year-old male with MMSE 20 and thus clinically suspected AD with 20 month disease duration had decreased $r$ CBF in the parietal cortex and the posterior cingulate cortex. The severity index, extent and rCBF ratio were Severity: 3.3, Extent: 77.96\%, Ratio: 5.6 respectively. 
Zoom: $\mid \times 2.5 \quad$ - $\sqrt{ }$ Label 2.0 , 6

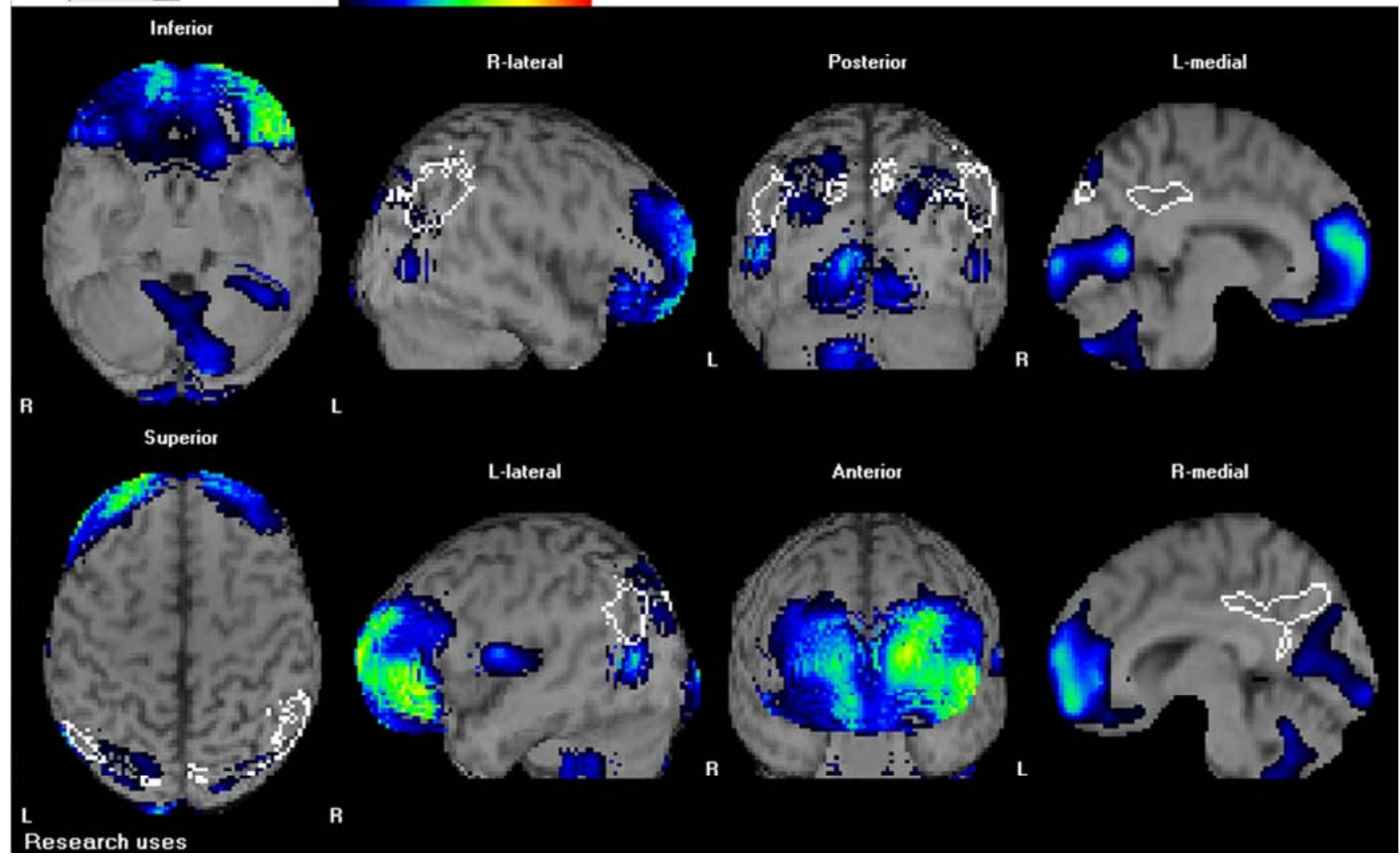

Figure 3. A 75-year-old female with clinically suspected FTD with a disease duration of 08 months exhibited a decreased $r C B F$ area in the frontal cortex activity. The severity index, extent and $r C B F$ ratio w ere $1.01(<1.19), 19.9 \%(<14.2 \%)$ and $1.90(<2.22)$, respectively.

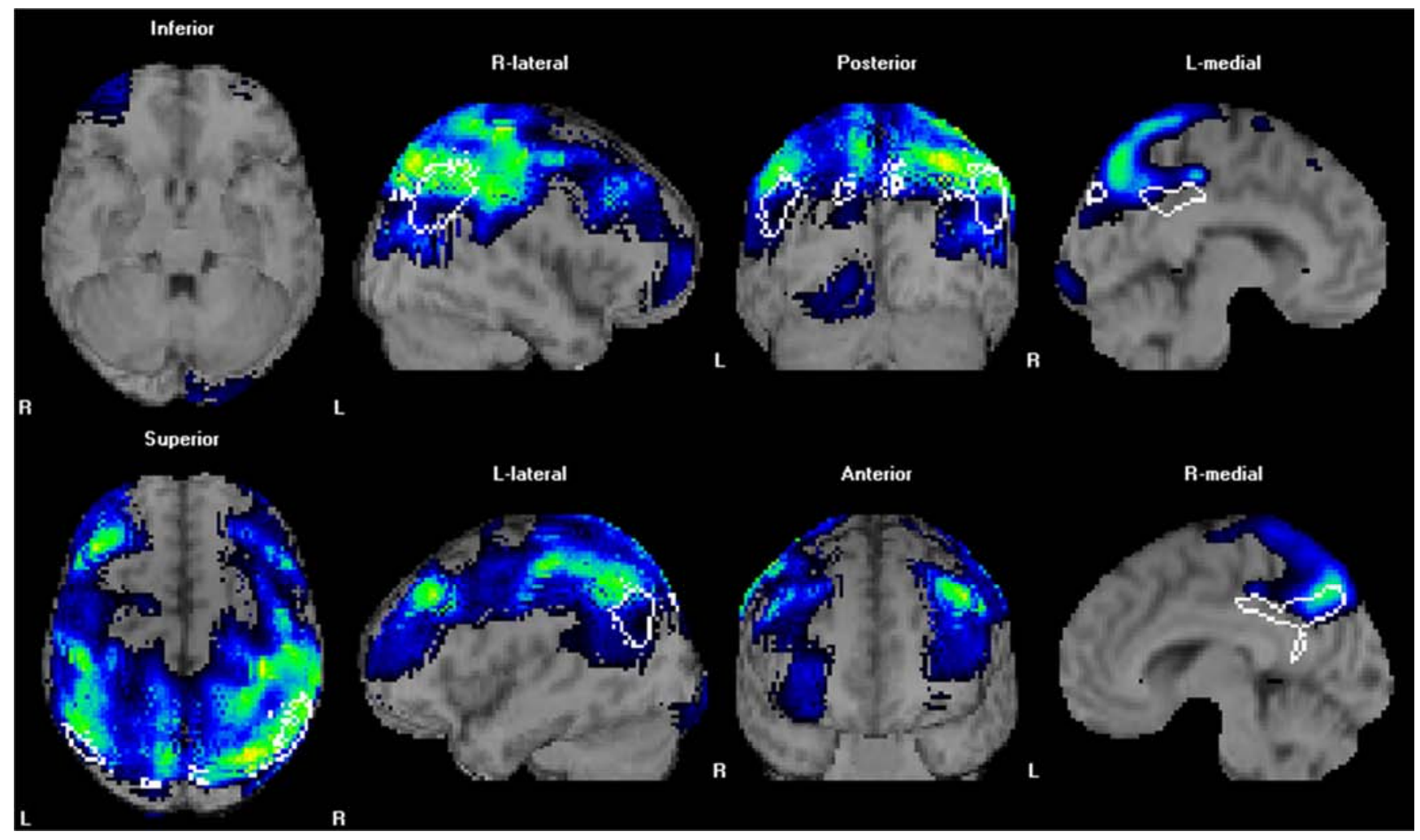

Figure 4. A 73-year-old male with clinically suspected AD, duration of 22 months exhibited a marked decreased $r C B F$ area in posterior cingulate gyri and parietal lobes may suggest moderate Alzheimer's disease. The severity index, extent and rCBF ratio were, Severity: 2.43 ( $<1.19)$, Extent: 56.50\% (<14.2\%), Ratio: $3.5(<2.2)$. 


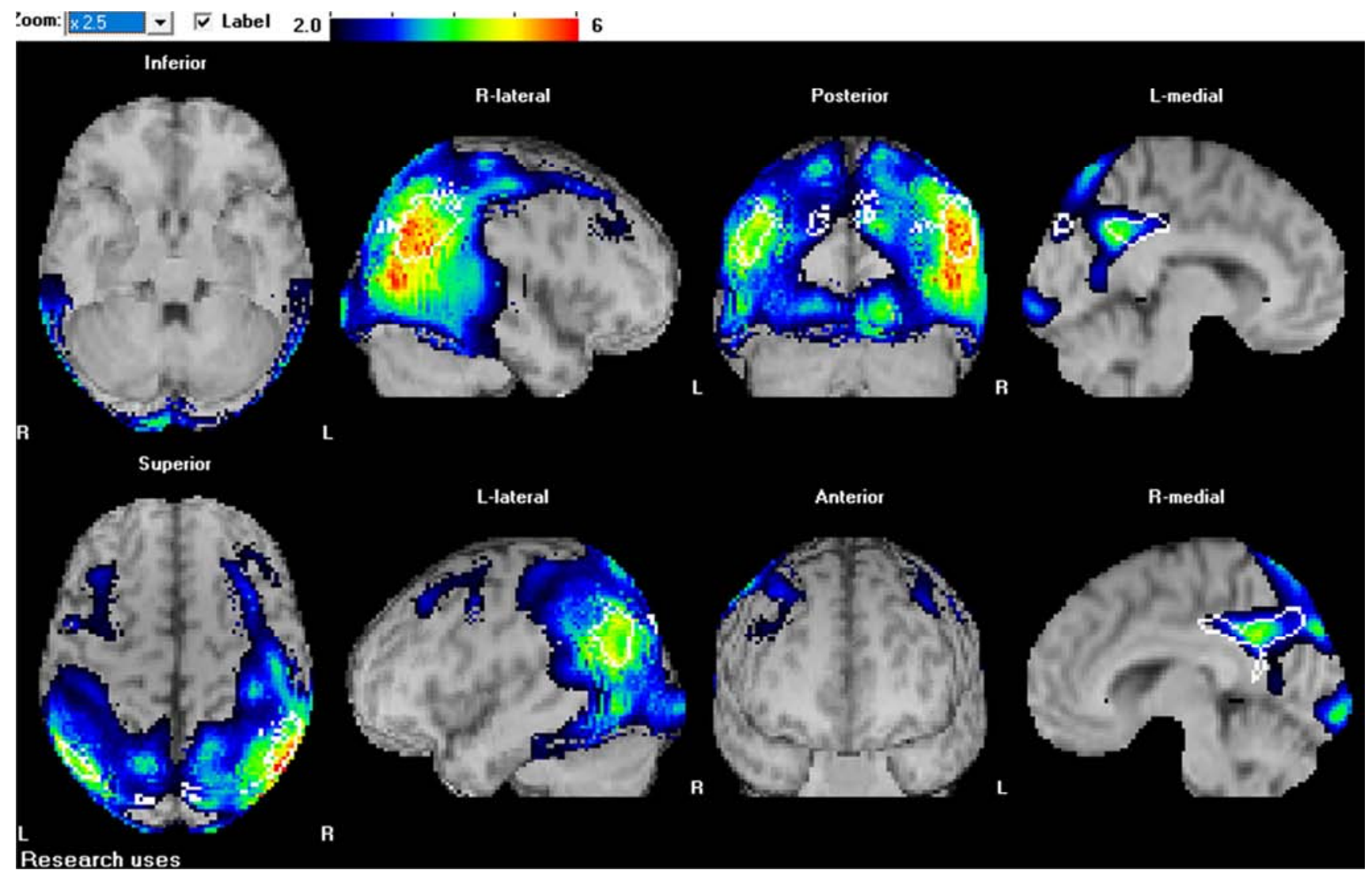

Figure 5. A $68 \mathrm{Y} / \mathrm{M}, \mathrm{MMSE}$ 18, with clinically suspected AD with disease duration of 20 months exhibited a marked decreased $r C B F$ area in occipital lobes, posterior cingulate gyri and parietal lobes may suggest dementia with Lewy body (DLB) along with moderate AD. The severity index, extent and $r C B F$ ratio were, Severity: $4(<1.19)$, Extent: $92.62 \%(<14.2 \%)$, Ratio: $5.6(<2.2)$.

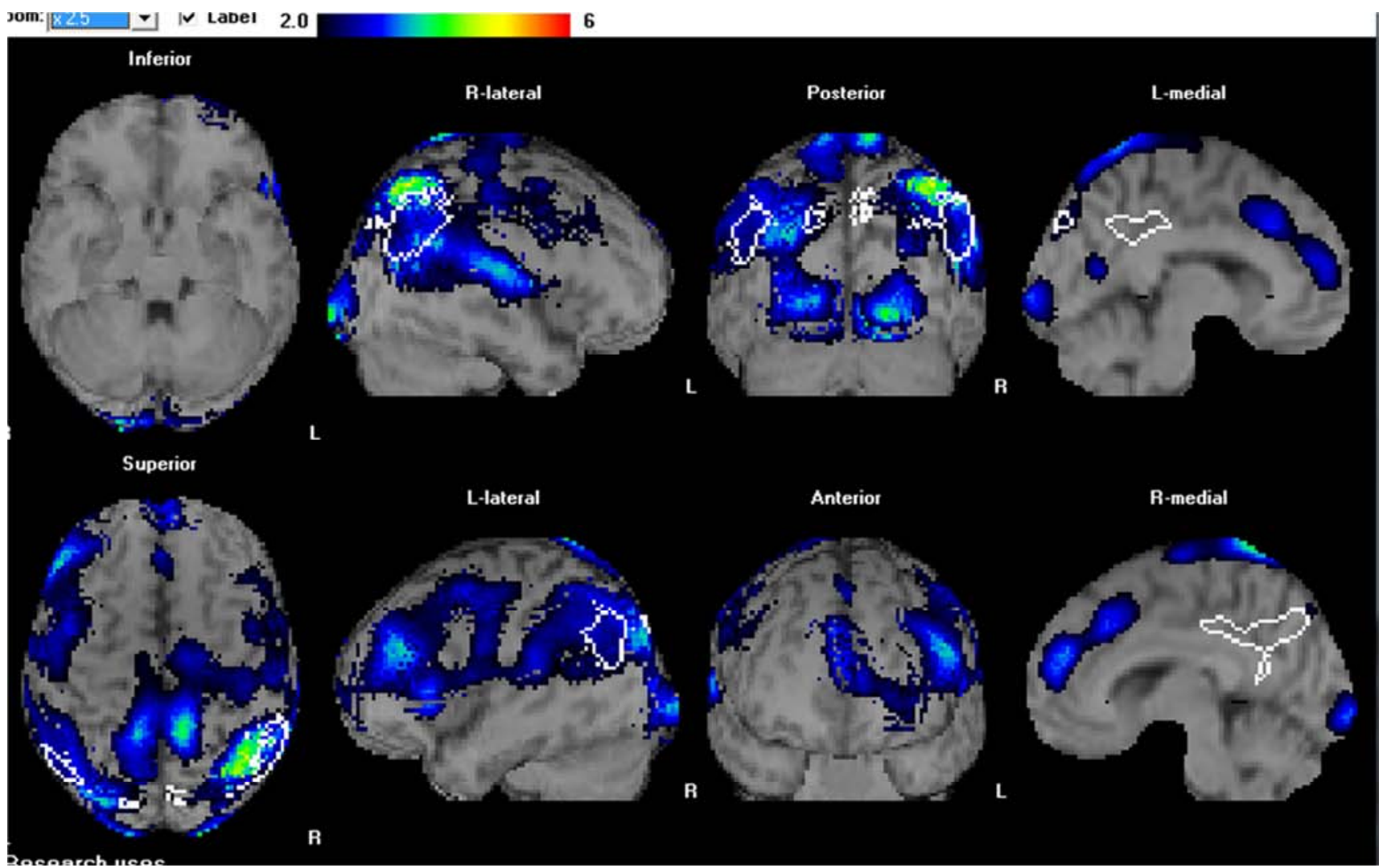

Figure 6. A 78-year-old male having history of CVA, clinically suspected VD patient with a 20-month disease duration had decreased rCBF in the basal ganglia, posterior cortex, occipital cortex and the cingulate cortex. The severity index, extent and rCBF ratio were 1.65, 19.36\% and 1.33 respectively. 


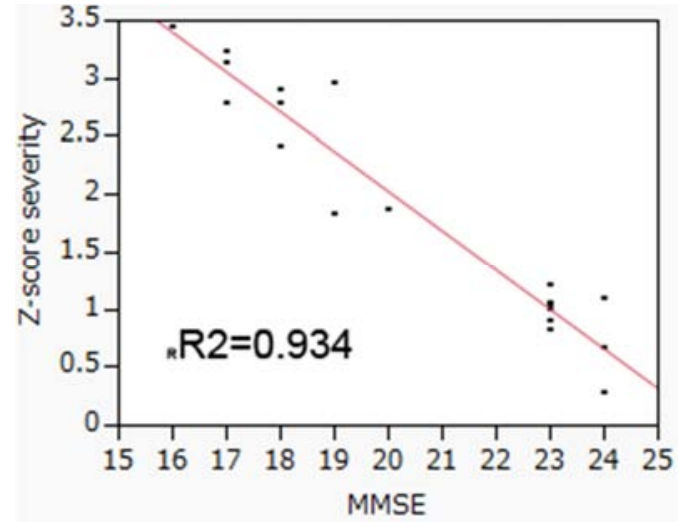

Figure 7. Scatter plot showing negative correlation between MMSE and ZScore severity in clinically suspected $A D(n=20)$ patients.

\section{Discussion}

In this study we tried to evaluate the differential diagnosis of neurodegenerative disease manifesting dementia. Decreased $\mathrm{rCBF}$ in the posterior cingulate gyrus and the precuneusis highly specific to early $\mathrm{AD}$ [8]. DLB is characterized by recurrent visual hallucinations, fluctuating cognitive impairment, and parkinsonism [11]. As recognized in $\mathrm{AD}$, this shows bilateral parieto-temporal dysfunction, DLB is associated with severe occipital hypometabolism and hypoperfusion in PET and SPECT images [9, 11, 12]. All clinically suspected DLB patients in the current study hadvisual hallucinations and mild cognitive impairment as initial symptoms, and eZIS analysis showed decreases in rCBF extending from the bilateral precuneus gyrus to the partial and occipital cortex. These findings seem to becompatible with neurodegenerative changes in DLB. Minoshima et al. reported high sensitivity (90\%) andspecificity $(80 \%)$ in discriminating AD from DLB based on hypometabolism in the occipital cortex [12]. The most common and early symptom of FTD is a decline in social interpersonal behavior [13]. SPECT or PET images show hypoperfusion of the anterior cerebral cortex with relative sparing of the posterior cortex in FTDpatients [14] using SPECT. We were able to demonstrate decreased $\mathrm{rCBF}$ in the frontal cortex, temporalcortex and the orbitofrontal cortex. These regionsare related to antisocial behavioral symptoms in patients with FTD [15]. Bonte et al, emphasized the presence of a decrease in $\mathrm{rCBF}$ in the posterior cingulate gyrus (posterior cingulate sign) for differentiating $\mathrm{AD}$ fromFTD [16].

Our results suggest that the rCBF pattern may be useful in differentiatingearly $\mathrm{AD}$ from other types of dementia. Our results show that the values of the $Z$ score severity may be usefulfor discriminating $\mathrm{AD}$ from other types of dementia as an adjunct to the early diagnosis of patients with dementia. Quantification $\mathrm{rCBF}$ in specific VOI with $\mathrm{Z}$ score severity were also well correlated with clinical MMSE scoring in this study group with clinical likelihoodof having AD.

\section{Conclusion}

Brain perfusion SPECT with eZIS has very important role in differential diagnosis of dementia. A specific rCBF pattern was observed for each disease using eZIS analysis that consistent with clinical complaint. eZIS indices, especially the $\mathrm{rCBF}$ pattern, may be useful in establishing the differential diagnosis between early-stage AD and FTD or VD. Quantification of rCBF with eZIS score was also well associated with clinical MMSE scoring in this study group with clinical likelihood of having AD. This was the first experiences of application of eZIS in brain perfusion SPECT imaging in single institute of Bangladesh. We recommend to use of eZIS as an unbiased adjunct to MMSE for clinicians to aid initiation of appropriate pharmacotherapy in patients with suspected $\mathrm{AD}$ and to differentiate the diagnosis of dementia with neurodegenerative disease.

\section{References}

[1] Ashburner J, Friston KJ: Voxel-based morphometry- the methods. Neuroimage 2000; 11: 805-821.

[2] Frith CD, Friston KJ, Ashburner J, et al: Principlesand methods; in Frackowiak RSJ, Friston KJ, Frith CD, Dolan RJ, Mazziotta JC (eds): Human Brain Function, ed 1. San Diego, Academic Press, 1997, pp 3-159.

[3] Minoshima S, Berger KL, Lee KS, et al: Anautomated method for rotational correction and centering of threedimensional functional brain images. J Nucl Med 1992; 33: 1579-1585.

[4] Minoshima S, Koeppe RA, Mintun MA, etal: Automated detection of the intercommissural line for stereotactic localization of functional brain images. J Nucl Med 1993; 34: 322-329.

[5] Minoshima S, Koeppe RA, Frey KA, et al: Anatomic standardization: linear scaling and nonlinear warping of functional brainimages. J Nucl Med 1994; 35: 1528-1537.

[6] Matsuda H, Mizumura S, Soma T, et al: Conversionof brain SPECT images between different collimators and reconstruction processes for analysis using statistical parametric mapping. Nucl Med Commun 2004; 25: 67-74.

[7] Kanetaka H, Matsuda H, Asada T, et al: Effects of partial volume correction on discrimination between very early Alzheimer's dementia and controls using brain perfusion SPECT. Eur J Nucl Med Mol Imaging 2004; 31: 975-980.

[8] Matsuda H, Mizumura S, Nagao T, et al: Automated discrimination between very early Alzheimer's disease and controls using an easy Z-score imaging system for multicenter brain perfusion SPECT. AJNR Am J Neuroradiol 2007; 28: $731-736$.

[9] Matsuda H: Role of neuroimaging in Alzheimer's disease, with emphasis on brain perfusion SPECT. J Nucl Med 2007; 48: 1289-1300. 
[10] Waragai M, Yamada T, Matsuda H: Evaluation of brain perfusion SPECT using an easy Z-score imaging system (eZIS) as an adjunct to early diagnosis of neurodegenerative diseases. J Neurol Sci 2007; 260: 57-64.

[11] McKeith IG, Boeve BF, Dickson DW, Halliday G, Taylor JP, Weintraub D, et al. Diagnosis and management of dementia with Lewy bodies: Fourth consensus report of the DLB Consortium. Neurology 2017; 89: 88-100.

[12] Minoshima S, Foster NL, Sima AA, et al: Alzheimer's disease versus dementia with Lewy bodies: cerebral metabolic distinction with autopsy confirmation. Ann Neurol 2001; 50: 358-365.

[13] Rosen HJ, Hartikainen KM, Jagust W, et al: Utility of clinical criteria in differentiating frontotemporal lobar degeneration (FTLD) from AD. Neurology 2002; 58: 1608-1615.
[14] Charpentier P, Lavenu I, Defebvre L, et al: Alzheimer's disease and frontotemporal dementia are differentiated by discriminant analysis applied to ${ }^{99 \mathrm{~m}} \mathrm{Tc}$ HmPAO SPECT data. J Neurol Neurosurg Psychiatry 2000; 69: 661-663.

[15] Nakano S, Asada T, Yamashita F, et al: Relationship between antisocial behavior and regional cerebral blood flow in frontotemporal dementia. Neuroimage 2006; 32: 301-306.

[16] Bonte FJ, Harris TS, Roney CA, et al: Differential diagnosis between Alzheimer's and frontotemporal disease by the posterior cingulatesign. J Nucl Med 2004; 45: 771-774. 